\title{
Child and Family \\ Factors Associated \\ With Child Maltreatment \\ in Vietnam
}

\author{
Nhu K. Tran,' Sheila R. van Berkel,' \\ Marinus H. van IJzendoorn, ${ }^{2}$ \\ and Lenneke R. A. Alink'
}

\begin{abstract}
This study aims to explore possible risk factors for child maltreatment in Vietnam by investigating the association of child and family factors with different types of child maltreatment (i.e., sexual abuse, physical abuse, emotional abuse, witnessing parental conflict, and neglect) and the occurrence of multiple types of child maltreatment. Cross-sectional data of $I, 85 I$ secondary and high school students aged 12 to 17 years $(47.3 \%$ boys) in four provinces of Northern Vietnam were obtained using selfreport questionnaires. Results indicated that the likelihood of emotional abuse, witnessing parental conflict, and experiencing multiple types of child maltreatment during lifetime increased with age. Boys had a higher risk than girls on lifetime sexual abuse, and past year and lifetime physical abuse. Living in a single parent family was the risk factor related to most types of child maltreatment including lifetime sexual abuse, neglect, and multiple types of child maltreatment, and both past year and lifetime witnessing parental conflict. Interestingly, low socioeconomic status (SES) and parental unemployment were associated with a decreased risk on experiencing emotional abuse in the past year and during lifetime, respectively. "Tiger
\end{abstract}

\footnotetext{
'Leiden University, The Netherlands

2Erasmus University Rotterdam, The Netherlands
}

Corresponding Author:

Lenneke R. A. Alink, Institute of Education and Child Studies, Leiden University, P.O. Box 9555, 2300 RB Leiden, Netherlands.

Email: alinklra@fsw.leidenuniv.nl 
parenting," a parenting style observed frequently in East Asian parents, may be more common in families with high SES and might explain this finding. This study highlights the importance of prioritizing single parent families in parenting programs and implementing child maltreatment interventions early because of the risk on child maltreatment increased with age. More research on emotional abuse and "Tiger parenting" in Vietnam could clarify the association of emotional abuse with high SES and parental employment. Finally, the underlying mechanisms of the risk factors in Vietnam should be studied more to inform interventions.

\section{Keywords}

child maltreatment, child abuse and neglect, risk factors, Vietnam

\section{Introduction}

Child maltreatment has negative consequences for individuals and societies at large (Fang et al., 2015; Tran, van Berkel, van IJzendoorn, \& Alink, 2017). Child maltreatment was found to be highly prevalent in Vietnam, with lifetime prevalence estimates for the different types ranging from $7.1 \%$ to $59.9 \%$ and past-year prevalence ranging from $2.6 \%$ to $31.8 \%$ (Tran, Alink, van Berkel, \& van IJzendoorn, 2017). Empirical inquiry on the risk factors for child maltreatment could provide scientific evidence for developing prevention and early intervention programs. However, most of the research on risk factors for child maltreatment was conducted in high income countries. Vietnam is a middle-income country with some specific characteristics such as high tolerance of harsh discipline and a long war history. Research on the risk factors for child maltreatment considering this specific context is very much needed. In this article, we explore risk factors for several types of child maltreatment separately as well as risk factors for having experienced multiple types of child maltreatment in Vietnam.

\section{Etiological Framework}

The etiological framework of child maltreatment by Belsky $(1980,1993)$ which was based on Bronfenbrenner's social ecological model (Bronfenbrenner, 1979) proposes that the occurrence of child maltreatment depends on factors at multiple subsystems in an interactive way. The model includes proximal factors including child and family factors and distal factors including social and cultural structures in which individuals and families are embedded. Proximal factors may affect child maltreatment more directly than distal factors. Therefore, the current study 
focuses on a number of proximal risk factors for child maltreatment in Vietnam: child age (within adolescence), gender, ethnic minority status, number of children in the family, single parenthood, parental unemployment, and socioeconomic status (SES). In addition, we will explore a risk factor related to the Vietnamese history: war veteran status of the father.

\section{Proximal Risk Factors for Child Maltreatment}

There is evidence that child age and gender are significantly related to the occurrence of one or more different types of child maltreatment. When zooming in on the period of adolescence, there is evidence for an increased risk of child maltreatment in general for older adolescents (Euser et al., 2013). Less is known about the association with age in adolescence for the specific types of maltreatment. Using data for a broader age range, meta-analytic results show that the risk for neglect and physical abuse appears to be unrelated to age (Stith et al., 2009). The risk for sexual abuse and emotional abuse seems to increase with age in samples ranging from 0 to 17 years of age (Black, Heyman, \& Smith Slep, 2001; Black, Smith Slep, \& Heyman, 2001). A systematic review of risk factors for intimate partner violence, which - in case this is witnessed by children - is considered a form of emotional abuse, showed that intimate partner violence occurs more often within younger couples, so younger children may be at increased risk for this type of emotional neglect (Capaldi, Knoble, Shortt, \& Kim, 2012). However, it is unknown whether this association also exists within adolescence.

Meta-analytic evidence shows that worldwide, the only gender difference is related to sexual abuse, with girls being more at risk than boys (Stoltenborgh, Bakermans-Kranenburg, Alink, \& van IJzendoorn, 2014). However, a metaanalysis on sexual abuse in China showed a higher risk among boys (Ji, Finkelhor, \& Dunne, 2013). The absence of gender differences for the other types of maltreatment is confirmed by several studies (Black, Heyman, \& Smith Slep, 2001; Black, Smith Slep, \& Heyman, 2001; Stith et al., 2009). In addition, ethnic minority status seems to be related to a number of maltreatment types. There is evidence that maltreatment occurs more often in families with a minority background as compared with other families (Alink, Euser, van IJzendoorn, \& Bakermans-Kranenburg, 2013; Black, Smith Slep, \& Heyman, 2001). However, these studies mostly report about minorities with a (recent) immigrant status. It is not known whether the same risk applies to minorities in Vietnam, whose immigrant status was not explored in this study.

Regarding family factors, several large prevalence studies have shown an increased risk of child maltreatment in families with low SES, unemployment, single parents, and large family size (Euser et al., 2013; Sedlak et al., 
2010). More specifically, low SES was shown to increase the risk of physical abuse, emotional abuse, neglect, and intimate partner violence as a proxy for witnessing parental conflict (Black, Smith Slep, \& Heyman, 2001; Capaldi et al., 2012; Stith et al., 2009). Single parenthood and parental unemployment were found to be associated with higher risk of sexual abuse, physical abuse, and neglect (Black, Heyman, \& Smith Slep, 2001; Stith et al., 2009). Parental unemployment also increased the risk of intimate partner violence (Capaldi et al., 2012). Large family size is another family risk factor related to physical abuse and neglect (Stith et al., 2009).

\section{Child Maltreatment in Vietnam}

Data from the current study revealed that all types of child maltreatment were highly prevalent in Vietnam, ranging from $2.6 \%$ for sexual abuse to $31.8 \%$ for emotional abuse. With the exception of sexual abuse, the prevalence of all types of child maltreatment in Vietnam was higher than in the Netherlands (Tran, Alink, et al., 2017). Similar as in other South East Asian countries, the saying "Spare the rod, spoil the child" describes the cultural norms on parenting in Vietnam that harsh discipline (emotionally and physically) is considered necessary to raise well-behaved children (Beazley, Bessell, Ennew, \& Waterson, 2006). Authoritarian parenting is common in Confucian cultures such as Vietnam (Ang \& Goh, 2006; Nguyen, 2008). Available research on parenting with Vietnamese/ Asian immigrants in developed countries suggests the existence of Tiger parenting, a practice of harsh discipline with the emphasis on study achievements and family obligations among Vietnamese/Asian parents (Kim, Wang, OrozcoLapray, Shen, \& Murtuza, 2013; Nguyen, Chang, \& Loh, 2014).

Another specific characteristic of Vietnam is its long war history. Irrespective of ethnic background, military service was compulsory in Vietnam for male residents from 18 to 45 years old. It is known that war exposure is related to psychiatric disorders such as depression and posttraumatic stress disorder (Leone, 2017; Miller \& Rasmussen, 2010), which in turn are related to dysfunctional parenting and child maltreatment (Smith, 2004; Stith et al., 2009). There is also some evidence for the direct association between war experience and maltreatment. For example, combat-related deployment has been associated with higher rates of child maltreatment in military families in the United States (Gibbs, Martin, Kupper, \& Johnson, 2007; Rentz et al., 2007) and a study in Uganda showed that the severity of posttraumatic stress disorder (PTSD) was associated with more child maltreatment reported by the veterans' children (Saile, Ertl, Neuner, \& Catani, 2014). The effect of parental war experience on child maltreatment in Vietnam has not been examined in previous research. 
One of the few studies on risk factors for child maltreatment in Vietnam (Nguyen, 2006) revealed several risk factors, such as a low family SES, gender, rurality, family composition, and employment. Unfortunately, these results have not been published, and they reflect the situation in Vietnam more than 10 years ago. In addition, a Vietnamese study on risk factors for intimate partner violence specifically found that lower education, a history of sexual violence, and experiences with child physical abuse were risk factors for women to be victims, and young age, alcohol use, extramarital relationships, and child abuse experience were risk factors for men to be perpetrators. Moreover, low SES was related to more intimate partner violence. Child gender, number of children in the family, ethnicity, and employment status were not related to intimate partner violence (Jansen, Nguyen, \& Hoang, 2016).

Clearly, most research on child maltreatment has been conducted in Western settings, in particular in North American and European societies (Stoltenborgh et al., 2014). Research on risk factors in limited resource settings is lacking. In addition, worldwide there is a wide range of literature exploring risk factors for sexual abuse and physical abuse but fewer studies focus on risk factors for emotional abuse, witnessing parental conflict, and neglect. A large number of studies did not examine all types of child maltreatment, and were thus unable to explore the risk factors for experiencing multiple types of child maltreatment. In addition, absence of multivariate analysis limits the possibility to control for the role of other risk factors when exploring a specific factor (H. T. Nguyen, 2006; Stith et al., 2009).

\section{Aim of the Current Study}

To address the limitations of previous research as well as to provide scientific evidence for preventive child maltreatment interventions in the context of high child maltreatment prevalence in a limited resource country, we studied risk factors for maltreatment in the cross-sectional "Vietnam Prevalence Study on Child Maltreatment" (VPM-2014) study using a representative student sample in Northern Vietnam (see also Tran, Alink, et al., 2017; Tran, van Berkel, et al., 2017). We used multivariate analyses to investigate the association between proximal factors on the level of the child (age, gender, ethnic minority) and the family (number of children in the family, single parenthood, parental unemployment, low SES, war veteran experience of father), and the various types of child maltreatment including sexual abuse, physical abuse, emotional abuse, witnessing parental conflict, neglect, and the occurrence of multiple types of child maltreatment to explore possible risk factors for child maltreatment in Vietnam. 


\section{Method}

\section{Sample}

The study was conducted in four provinces of Northern Vietnam, namely Hanoi, Nam Dinh, Ha Tinh, and Tuyen Quang. Hanoi, the capital of Vietnam, was selected because of its unique metropolitan characteristics. Next, one province was randomly selected from each of the three geographic areas of Northern Vietnam. In each province, two secondary schools and two high schools were randomly selected. In Hanoi, only schools in urban areas were selected because this province has the largest metropolitan population. In each of the other three provinces, one secondary school (for children aged 12 to 14 years) was selected from a list of schools in urban areas and the other secondary school from a list of schools in rural areas. In addition, for logistical reasons, for each province, the two high schools (for children aged 15 to 17 years) that were nearest to the secondary schools were selected. Exclusion criteria included schools for blind students, schools with fewer than 40 students per grade, and boarding schools where children live full time. One or two classes per grade of each participating school, depending on the number of students in a class, were randomly selected (Tran, Alink, et al., 2017; Tran, van Berkel, et al., 2017).

Our sample thus consisted of a total of eight secondary schools and eight high schools. In total, 2,360 students from 71 classes participated in the study. Students were excluded when unreliable answers were suspected based on outlying scores (more than 3.29 SD above the mean; Tabachnick \& Fidell, 2012) on the Wildman Symptom Checklist, a scale consisting of bogus symptoms, such as "The buzzing in my ears keeps switching from the left to the right" (Merckelbach, Smeets, \& Jelicic, 2009; Wildman \& Wildman, 1999; $n=31$ ), or based on a specific pattern in their answers on the maltreatment questionnaire (e.g., all questions answered with the highest possible score; $n=23$ ). In addition, students who were older than $17(n=331)$ or younger than 12 years $(n=2)$ or with missing data concerning age $(n=123)$ were excluded. The final sample consisted of 1,851 students $(47.3 \%$ boys, $57.6 \%$ secondary school students; Table 1 ). The students were equally distributed among the four provinces. Most of the students were Kinh (81.7\%), which is the majority ethnic group of Vietnam, and $17.8 \%$ belonged to one of the ethnic minority groups (the other $0.5 \%$ had missing values for ethnicity). The mean age of the students was 14.2 years $(S D=1.4)$.

\section{Procedure}

After the Provincial Department of Education and the school boards approved the implementation of the study in the schools, passive informed consent was 
Table I. Sample Description $(N=1,85 \mathrm{I})$.

\begin{tabular}{lcc}
\hline Variable & $N$ & $\%$ \\
\hline Gender & & \\
Boys & 875 & 47.3 \\
Girls & $96 \mathrm{I}$ & 51.9 \\
School & 1,066 & \\
$\quad$ Secondary schools & 785 & 57.6 \\
High schools & 1,512 & 42.4 \\
Ethnicity & 329 & 81.7 \\
Major ethnicity & $M$ & 17.8 \\
Minor ethnicity & 14.2 & $S D$ \\
\hline & & 1.4 \\
\hline Age & & \\
\hline
\end{tabular}

Note. $0.8 \%$ of sample missing on gender; $0.5 \%$ of the sample missing on ethnicity.

obtained from both the students and their parents. The students filled out the questionnaire during class hours. Students who refused or students whose parents refused participation in the study filled out dummy questionnaires to avoid making these students a special group in the classroom. These questionnaires were destroyed after the study and were thus not used in our analyses. The students were provided with contact details of the researcher and of the Child Protection Helpline for consultation and support. The Child Protection Helpline, the free hotline of Ministry of Labour, Invalids and Social Affairs, provides consultation, referral, and support for child abuse and neglect cases. The research proposal was approved by the Ethics Committee of the Institute of Education and Child Studies of Leiden University and the Ethics Committee of the Institute of Population, Health and Development of Vietnam.

\section{Measures}

Child maltreatment. The child maltreatment questionnaire (see also Euser et al., 2013) contained 32 items and was based on the Dating Violence Questionnaire (Douglas \& Straus, 2006) and the Parent-Child Conflict Tactics Scales (CTSPC; Straus, Hamby, Finkelhor, Moore, \& Runyan, 1998). The scale consists of the following subscales: Emotional Abuse (one item), Physical Abuse (eight items), Witnessed Parental Conflict (seven items), Sexual Abuse (eight items), and Neglect (eight items). The maltreatment items were 
embedded in a questionnaire with filler items, which concerned unpleasant and nasty incidents, nonviolent parental discipline (CTSPC; Straus et al., 1998), and the social desirability items of the Dating Violence Questionnaire (Douglas \& Straus, 2006). The Cronbach's alpha of the whole maltreatment scale was .75. In addition, the Cronbach's alphas of the Child Maltreatment subscales were also adequate ( $\alpha \mathrm{s} \geq .69$ ). We also computed the sum of all types of child maltreatment during the past year and lifetime, respectively (for neglect only lifetime experience was available), so a higher score indicates that the participant had experienced more types of child maltreatment.

Child and family risk factors. Participants were asked to report their age, gender, ethnicity, and the number of children living with them in the family. In addition, they were asked about the type of family they were living in (e.g., two parents, single parent, with relatives, in a boarding school), the unemployment status of each parent, the educational level of their parents (never went to school, primary school, secondary school, high school, college/university or higher), items their family owned (such as a color television, fridge, mobile phone, etc.), and whether or not their father had served in the army during the war. Regarding living situation, $89.8 \%$ of the students lived with two parents, $6.7 \%$ lived in single parent families, and $3.5 \%$ had other living situations. We first focused on single parent families versus two-parent families, and repeated all analyses with the variable representing single parent families versus all other families. Concerning unemployment status, parents without an income from a job were considered unemployed. Parental unemployment was computed as the sum of the unemployment status of father and mother $(0=$ neither of the parents unemployed, 1 = one parent unemployed, $2=$ both parents unemployed). For single parent families, parental unemployment was defined using only the unemployment status of the parent that the child lived with. Finally, SES was computed as the standardized sum of the metropolitan level of the living area (urban vs. rural), the parents' level of education, and the number of items in the household. The combination of these three variables was based on the results of a principal components analysis, indicating that a one-factor solution explained $65 \%$ of the variance and factor loadings were high (metropolitan level of the living area: 0.71; parental education: 0.80, number of items in household: 0.87 ). Lower SES was indicated by a rural living area, low parental education, and fewer items in the household.

\section{Statistical Procedures}

As missing values were not missing completely at random (MCAR) and the percentage of missing values ranged from $0.3 \%$ (neglect) to $19.8 \%$ (having a 
war veteran father), we imputed the missing values using all study variables. Multiple imputation was performed in SPSS 23 using all background, predictor, and outcome variables (van Buuren, 2012). To deal with nonnormal distribution and outliers, the variable "number of children in the family" was transformed using a $\log 10$ transformation and winsorized before imputing missing data. One hundred data sets were imputed to allow for detection of small effect sizes even with variables with a rather high percentage of missing values, as in the case of having a war veteran father (Graham, Olchowski, \& Gilreath, 2007).

To explore the relation between risk factors and child maltreatment, logistic regression analyses with each type of child maltreatment as outcome variable and child and family factors as predictors were conducted. Separate models were tested for past year and lifetime maltreatment. Results are based on the pooled standardized regression coefficients of the 100 imputed data sets and the pooled explained variance that was computed by calculating the mean (pseudo) $R^{2}$ of the 100 imputed data sets. Because of the number of tests, we used a conservative significance level of alpha $<.01$.

\section{Results}

\section{Bivariate Associations Between Variables}

The positive correlations between most types of maltreatment, both past year and lifetime, indicate modest comorbidity among types of child maltreatment (Table 2). All maltreatment variables were significantly intercorrelated for past year and lifetime experiences with a few exceptions. Lifetime emotional abuse was negatively associated with neglect and was not associated with both past year and lifetime sexual abuse and witnessing parental conflict. Past year emotional abuse was not related to lifetime experience of sexual abuse and neglect.

Based on the correlation matrix, multicollinearity among child and family factors was not an issue (the maximum $r=.43$ ). Child age was positively related to lifetime emotional abuse, witnessing parental conflict, and the number of child maltreatment types a child experienced. In addition, boys were more likely to have experienced lifetime sexual abuse and physical abuse and to have experienced more types of child maltreatment during the past year than girls. Belonging to an ethnic minority was positively associated with lifetime sexual abuse and negatively with lifetime physical abuse. Living in a single parent family was positively related to lifetime sexual abuse, neglect, witnessing parental conflict, and the number of experienced child maltreatment types both past year and lifetime. Parental unemployment was negatively associated 


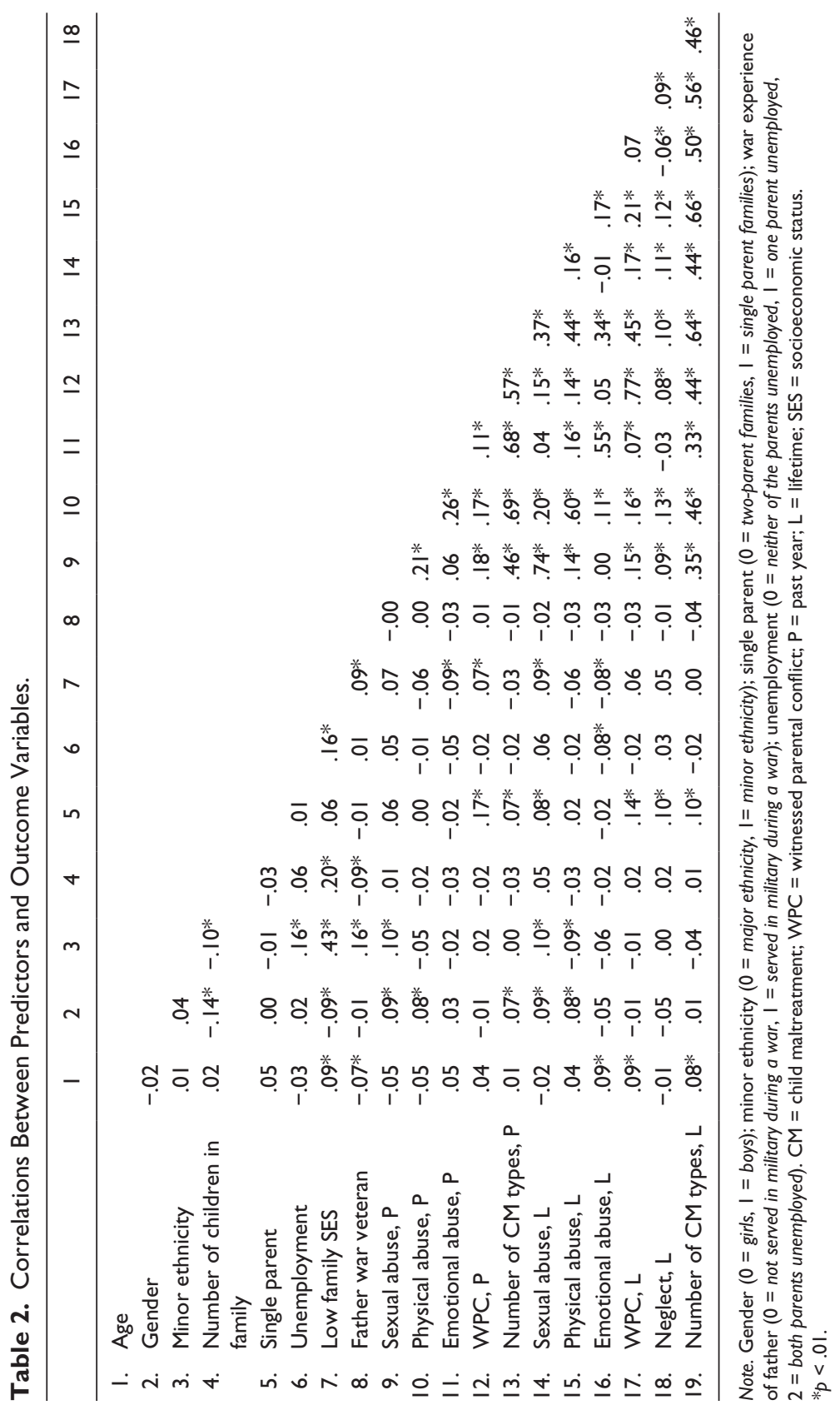


Table 3. Results of the Logistic Regression Models Predicting Sexual Abuse.

\begin{tabular}{|c|c|c|c|c|c|c|c|c|}
\hline & \multirow{2}{*}{\multicolumn{4}{|c|}{$\begin{array}{c}\text { Sexual Abuse Past Year } \\
\text { Nagelkerke } R^{2}=.05\end{array}$}} & \multirow{2}{*}{\multicolumn{4}{|c|}{$\begin{array}{c}\text { Sexual Abuse Lifetime } \\
\text { Nagelkerke } R^{2}=.05\end{array}$}} \\
\hline & & & & & & & & \\
\hline & B & $S E$ & $p$ & $\begin{array}{l}\text { Odds } \\
\text { Ratio }\end{array}$ & B & $S E$ & $p$ & $\begin{array}{l}\text { Odds } \\
\text { Ratio }\end{array}$ \\
\hline Age & -.12 & .09 & .168 & 0.89 & -.02 & .06 & .786 & 0.98 \\
\hline Gender & .68 & .27 & .011 & 1.98 & .65 & .19 & .001 & $1.92 *$ \\
\hline Ethnic minority & .53 & .37 & .151 & 1.69 & .55 & .27 & .046 & 1.73 \\
\hline $\begin{array}{l}\text { Number of children in } \\
\text { family }\end{array}$ & .48 & .56 & .392 & 1.62 & .93 & .42 & .026 & 2.55 \\
\hline Single parent $\mathrm{t}^{\mathrm{a}}$ & .59 & .41 & .146 & 1.81 & .75 & .29 & .009 & $2.12^{*}$ \\
\hline Parental unemployment & .20 & .23 & .383 & 1.22 & .24 & .17 & .153 & 1.27 \\
\hline $\begin{array}{l}\text { Low social economic } \\
\text { status }\end{array}$ & .02 & .03 & .459 & 1.02 & .01 & .02 & .551 & I.0I \\
\hline Father war veteran & -.10 & .30 & .727 & 0.90 & -.17 & .21 & .406 & 0.84 \\
\hline
\end{tabular}

Note. Gender ( 0 = girls, I = boys $)$.

aSingle parent families vs. two-parent families; we also conducted the logistic regression with single parent families versus all other families and the results are similar (sexual abuse past year: odds ratio $=2.06, p=.072$; sexual abuse lifetime: odds ratio $=2.32, p=.003$ ). $*_{p}<.0 \mathrm{l}$.

with emotional abuse during lifetime. Lower SES was associated with more past year witnessing parental conflicts and more lifetime sexual abuse, and with less emotional abuse both past year and lifetime.

\section{Multivariate Associations Between Child and Family Factors and Maltreatment}

The results of the logistic regression models predicting sexual abuse are described in detail in Table 3. Boys were more likely to have experienced sexual abuse during lifetime compared with girls, odds ratio $(\mathrm{OR})=1.92$, $95 \%$ confidence interval $(\mathrm{CI})=[1.32,2.78]$. Similarly, living in a single parent family was associated with a higher risk of experiencing lifetime sexual abuse $(\mathrm{OR}=2.12,95 \% \mathrm{CI}=[1.20,3.74])$.

The odds of being physically abused, both in the past year and during lifetime, were higher for boys than for girls (Table $4 ; \mathrm{OR}=1.46,95 \% \mathrm{CI}=[1.13,1.89]$, for past year; $\mathrm{OR}=1.46,95 \% \mathrm{CI}=[1.20,1.78]$, for lifetime). Furthermore, both past year and lifetime physical abuse were not associated with child age or any of the family factors. 
Table 4. Results of the Logistic Regression Models Predicting Physical Abuse.

\begin{tabular}{|c|c|c|c|c|c|c|c|c|}
\hline & \multirow{2}{*}{\multicolumn{4}{|c|}{$\frac{\text { Physical Abuse Past Year }}{\text { Nagelkerke } R^{2}=.02}$}} & \multirow{2}{*}{\multicolumn{4}{|c|}{$\begin{array}{c}\text { Physical Abuse Lifetime } \\
\text { Nagelkerke } R^{2}=.02\end{array}$}} \\
\hline & & & & & & & & \\
\hline & $B$ & $S E$ & $p$ & $\begin{array}{l}\text { Odds } \\
\text { Ratio }\end{array}$ & $B$ & $S E$ & $p$ & $\begin{array}{l}\text { Odds } \\
\text { Ratio }\end{array}$ \\
\hline Age & -.07 & .05 & .122 & 0.93 & .07 & .04 & .070 & 1.07 \\
\hline Gender & .38 & .13 & .003 & $1.46 *$ & .38 & .10 & .000 & $1.46 *$ \\
\hline Ethnic minority & -.27 & .22 & .216 & 0.77 & -.38 & .17 & .021 & 0.68 \\
\hline Number of children in family & .03 & .31 & .931 & 1.03 & -.03 & .25 & .914 & 0.97 \\
\hline Single parent ${ }^{\mathrm{a}}$ & -.06 & .24 & .816 & 0.94 & .03 & .20 & .875 & 1.03 \\
\hline Parental unemployment & .01 & .15 & .969 & 1.01 & -.02 & .12 & .862 & 0.98 \\
\hline Low social economic status & -.01 & .01 & .398 & 0.99 & .00 & .01 & .840 & 1.00 \\
\hline Father war veteran & .01 & .15 & .944 & 1.01 & -.02 & .12 & .861 & 0.98 \\
\hline
\end{tabular}

Note. Gender $(0=$ girls, I = boys $)$.

aSingle parent families vs. two-parent families; we also conducted the logistic regression with single parent versus all other families and the results are similar (physical abuse past year: odds ratio $=1.04, p=.872$; physical abuse lifetime: odds ratio $=1.14, p=.528$ ).

$*_{p}<.0 \mathrm{I}$.

Regarding emotional abuse, child age was the only child characteristic that was significantly related to emotional abuse (Table 5). The odds of emotional abuse during lifetime increased with age $(\mathrm{OR}=1.12,95 \% \mathrm{CI}=[1.04,1.22])$. With respect to family factors, lower SES was significantly associated with less emotional abuse in the past year $(\mathrm{OR}=0.96,95 \% \mathrm{CI}=[0.94,0.98])$ and parental unemployment was related with less emotional abuse during lifetime $(\mathrm{OR}=0.72,95 \% \mathrm{CI}=[0.57,0.91])$.

The logistic regression analysis predicting witnessing parental conflicts showed that child age was the only child factor that was positively associated with lifetime experience of witnessing parental conflicts (Table 6; OR=1.14, $95 \% \mathrm{CI}=[1.05,1.23])$. Among the family factors, the odds of witnessing parental conflicts were higher for past year experiences (OR $=3.45,95 \%$ $\mathrm{CI}=[2.29,5.22])$ and for lifetime experiences among children living in single parent families $(\mathrm{OR}=2.51,95 \% \mathrm{CI}=[1.70,3.71])$.

For lifetime experience of neglect, the regression analysis only showed an effect of living situation (Table 7). Children living in single parent families were more likely to experience neglect $(\mathrm{OR}=2.09,95 \% \mathrm{CI}=[1.42,3.10])$. None of the child factors and other family factors were associated with neglect.

Finally, the number of child maltreatment types that children experienced during lifetime increased with age (Table 8). In addition, controlling for other 
Table 5. Results of the Logistic Regression Models Predicting Emotional Abuse.

\begin{tabular}{|c|c|c|c|c|c|c|c|c|}
\hline & \multirow{2}{*}{\multicolumn{4}{|c|}{$\begin{array}{c}\text { Emotional Abuse Past } \\
\text { Year }\end{array}$}} & \multirow{2}{*}{\multicolumn{4}{|c|}{$\begin{array}{c}\begin{array}{c}\text { Emotional Abuse } \\
\text { Lifetime }\end{array} \\
\text { Nagelkerke } R^{2}=.02\end{array}$}} \\
\hline & & & & & & & & \\
\hline & B & $S E$ & $p$ & $\begin{array}{l}\text { Odds } \\
\text { Ratio }\end{array}$ & B & $S E$ & $p$ & $\begin{array}{l}\text { Odds } \\
\text { Ratio }\end{array}$ \\
\hline Age & .08 & .04 & .037 & 1.09 & .12 & .04 & .002 & $1.12 *$ \\
\hline Gender & .07 & .11 & .520 & 1.07 & -.24 & .10 & .019 & 0.78 \\
\hline Ethnic minority & .24 & .17 & .143 & 1.28 & .01 & .16 & .938 & 1.01 \\
\hline Number of children in family & -.05 & .27 & .861 & 0.95 & -.12 & .25 & .632 & 0.89 \\
\hline Single parent ${ }^{\mathrm{a}}$ & -.18 & .21 & .399 & 0.83 & -.16 & .20 & .424 & 0.85 \\
\hline Parental unemployment & -.19 & .13 & .147 & 0.82 & -.33 & .12 & .004 & $0.72 *$ \\
\hline Low social economic status & -.04 & .01 & .004 & $0.96 *$ & -.02 & .01 & .074 & 0.98 \\
\hline Father war veteran & -.12 & .13 & .328 & 0.88 & -.03 & .12 & .807 & 0.97 \\
\hline
\end{tabular}

Note. Gender ( 0 = girls, I = boys $)$.

aSingle parent families vs. two-parent families; we also conducted the logistic regression with single parent versus all other families and the results are similar (emotional abuse past year: odds ratio $=0.85, p=.444$; emotional abuse lifetime: odds ratio $=0.83, p=.363$ ). $*_{p}<.01$.

family and child factors, living in a single parent family was associated with experiencing more types of child maltreatment during lifetime and the past year (but only when comparing with living in any other type of family). Figure 1 summarizes the results of all child and family risk factors associated with the different types of child maltreatment.

\section{Discussion}

This study examined the association between all specific types of child maltreatment and proximal risk factors in Vietnam. Results indicated that child gender and age were child factors related to several types of child maltreatment. In addition, living in a single parent family was the most frequent family factor associated with child maltreatment, followed by low SES and parental unemployment. In contrast, child ethnicity, the number of children in the family, and war veteran status of the father were not associated with any type of child maltreatment when potential confounders were controlled for.

Specifically, controlling for the other factors, age (12-17 years) was the child factor that was most frequently associated with child maltreatment. The likelihood of emotional abuse, witnessing parental conflict, and multiple 
Table 6. Results of the Logistic Regression Models Predicting Witnessing Parental Conflict.

\begin{tabular}{|c|c|c|c|c|c|c|c|c|}
\hline & \multirow{2}{*}{\multicolumn{4}{|c|}{$\begin{array}{l}\text { Witnessed Parental } \\
\text { Conflicts Past Year }\end{array}$}} & \multirow{2}{*}{\multicolumn{4}{|c|}{$\begin{array}{c}\text { Witnessed Parental } \\
\text { Conflicts Lifetime }\end{array}$}} \\
\hline & & & & & & & & \\
\hline & B & $S E$ & $p$ & $\begin{array}{l}\text { Odds } \\
\text { Ratio }\end{array}$ & B & $S E$ & $p$ & $\begin{array}{l}\text { Odds } \\
\text { Ratio }\end{array}$ \\
\hline Age & .06 & .05 & .282 & 1.06 & .13 & .04 & .003 & $1.14 *$ \\
\hline Gender & -.11 & .14 & .450 & 0.90 & -.10 & .12 & .403 & 0.91 \\
\hline Ethnic minority & -.09 & .21 & .679 & 0.92 & -.21 & .18 & .262 & 0.81 \\
\hline Number of children in family & -.37 & .36 & .313 & 0.69 & .02 & .30 & .951 & 1.02 \\
\hline Single parent ${ }^{\mathrm{a}}$ & 1.24 & .21 & .000 & $3.45^{*}$ & .92 & .20 & .000 & $2.51 *$ \\
\hline Parental unemployment & -.14 & .17 & .428 & 0.87 & -.11 & .14 & .427 & 0.89 \\
\hline Low social economic status & .03 & .02 & .119 & 1.03 & .02 & .01 & .075 & 1.02 \\
\hline Father war veteran & .15 & .17 & .395 & 1.16 & -.09 & .14 & .539 & 0.92 \\
\hline
\end{tabular}

Note. Gender $(0=$ girls, I = boys $)$.

aSingle parent families vs. two-parent families; we also conducted the logistic regression with single parent families versus all other families and the results are similar (witnessed parental conflicts past year: odds ratio $=3.87, p=.00$; witnessed parental conflicts lifetime: odds ratio $=2.78, p=.00)$.

$*_{p}<.01$.

Table 7. Results of the Logistic Regression Models Predicting Neglect.

\begin{tabular}{lcccc}
\hline & \multicolumn{4}{c}{ Neglect Lifetime } \\
\cline { 2 - 5 } & \multicolumn{4}{c}{ Nagelkerke $R^{2}=.02$} \\
\cline { 2 - 5 } Variable & $B$ & $S E$ & $P$ & Odds Ratio \\
\hline Age & -.02 & .04 & .637 & 0.98 \\
Gender & -.23 & .12 & .050 & 0.80 \\
Ethnic minority & -.18 & .18 & .319 & 0.83 \\
Number of children in family & .22 & .29 & .431 & 1.25 \\
Single parent & .74 & .20 & .000 & $2.09 *$ \\
Parental unemployment & .17 & .13 & .190 & 1.18 \\
Low social economic status & .01 & .01 & .270 & 1.01 \\
Father war veteran & -.08 & .14 & .591 & 0.93 \\
\hline
\end{tabular}

Note. Gender ( 0 = girls, I = boys $)$.

asingle parent families vs. two-parent families; we also conducted the logistic regression with single parent families versus all other families and the results are similar (odds ratio $=2.21$ and $p=.000$ ).

$*_{p}<.01$. 
Table 8. Results of the Regression Models Predicting Number of Maltreatment Types.

\begin{tabular}{|c|c|c|c|c|}
\hline & \multirow{2}{*}{\multicolumn{2}{|c|}{$\begin{array}{l}\text { Number of Types of } \\
\text { Child Maltreatment } \\
\text { Past Year }\end{array}$}} & \multirow{2}{*}{\multicolumn{2}{|c|}{$\begin{array}{c}\begin{array}{c}\text { Number of Types of } \\
\text { Child Maltreatment } \\
\text { Lifetime }\end{array} \\
R^{2}=.02\end{array}$}} \\
\hline & & & & \\
\hline & $\beta$ & $p$ & $\beta$ & $p$ \\
\hline Age & .01 & .719 & .06 & $.003^{*}$ \\
\hline Gender & .10 & .028 & .03 & .582 \\
\hline Ethnic minority & .04 & .581 & -.10 & .275 \\
\hline Number of children in family & -.02 & .881 & .10 & .473 \\
\hline Single parent ${ }^{\mathrm{a}}$ & .21 & .017 & .40 & $.000^{*}$ \\
\hline Parental unemployment & -.04 & .473 & -.04 & .509 \\
\hline Low social economic status & -.01 & .315 & .00 & .713 \\
\hline Father war veteran & -.02 & .777 & -.06 & .412 \\
\hline
\end{tabular}

Note.Gender ( 0 = girls, I = boys $)$.

aSingle parent families vs. two-parent families; we also conducted the logistic regression with single parent families versus all other families. And the results are similar for number of types of child maltreatment lifetime: unstandardized $\beta=.47, p=.000$. For number of types of child maltreatment past year, single parents became significant: unstandardized $\beta=.27, p=.004$. $*_{p}<.01$.

types of child maltreatment experience during lifetime increased with age, but we did not find significant associations between age and past year experience of any type of child maltreatment. A possible explanation for this is that as children grow older, they have had more experiences in general and possibly also more experiences of child maltreatment.

In our study, child gender was also frequently related to child maltreatment. Boys were at higher risk than girls for sexual abuse during lifetime and physical abuse during past year and lifetime, similar to the finding in a meta-analysis on child sexual abuse in China (Ji et al., 2013). It could be that sexual abuse among boys in the East Asian culture is indeed higher than that among girls. However, the taboo for girls to lose virginity before marriage in the East Asia culture, to some extent, may induce reluctance to report sexual abuse among girls. More studies on child sexual abuse in East Asia could clarify this concern. The finding that boys were at higher risk of physical abuse than girls was inconsistent with the meta-analysis of Stith et al. (2009) with studies available internationally on risk factors in child maltreatment which showed child gender not related to physical abuse. However, research on child maltreatment is mostly conducted in high-income countries, rather than in limited resource 


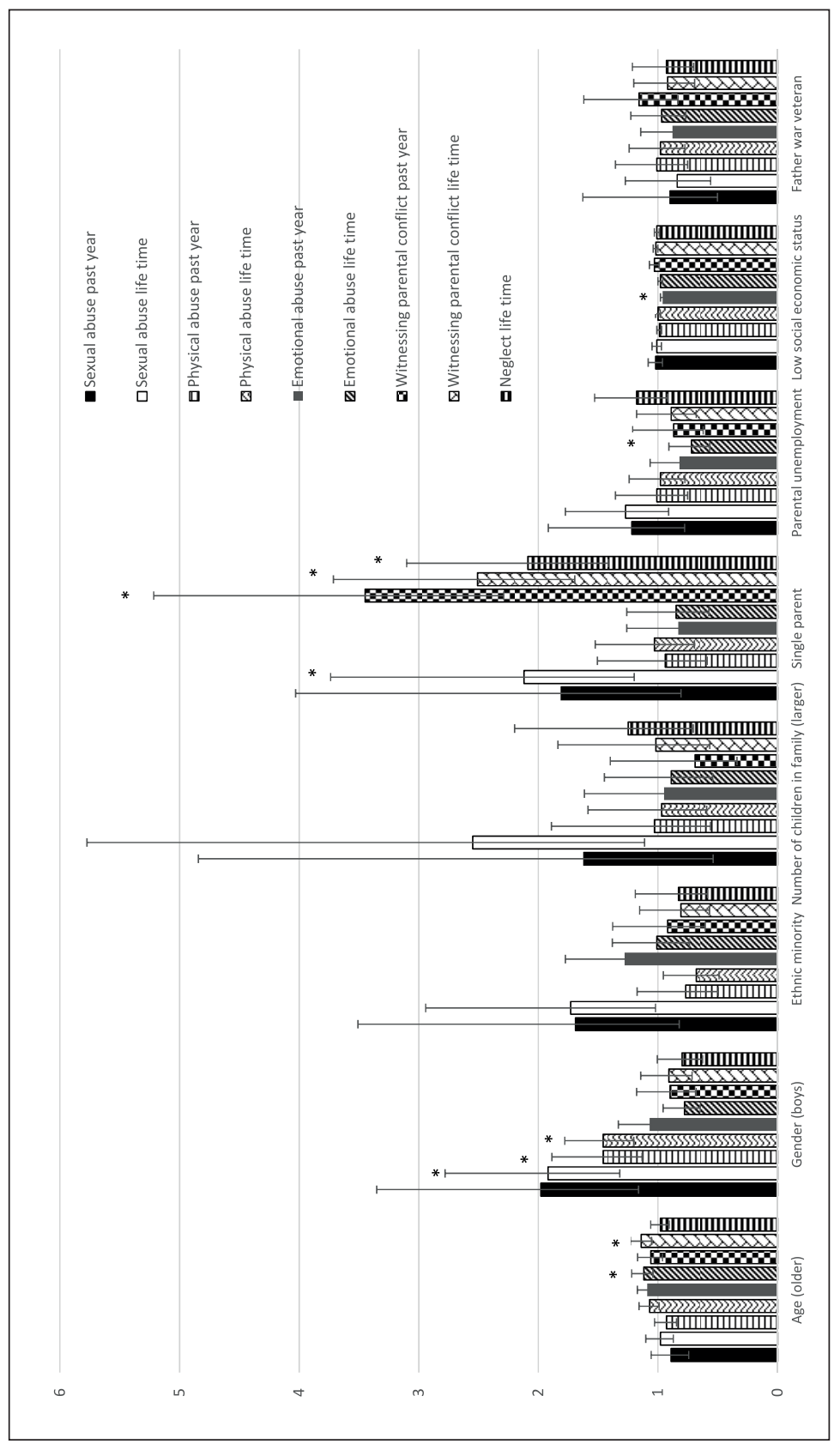

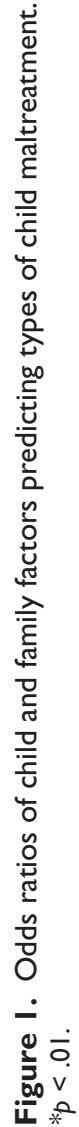


contexts (Mikton \& Butchart, 2009). In Vietnam and other similar East Asian cultures, there is a saying "Spare the rod, spoil the child." In addition, boys are generally preferred to girls (Guilmoto, 2012), so the harsher behaviors of parents with boys might express their greater focus on boys.

Among family factors, living in a single parent family was related to more sexual abuse, neglect, and the number of types of child maltreatment experience during lifetime, and witnessing parental conflict during both past year and lifetime. The finding that living in a single parent family increased the risk of sexual abuse during lifetime is in line with a review of studies based on U.S. samples (Black, Heyman, \& Smith Slep, 2001). There might be a lack of adequate supervision of children in single parent families, which may provide more opportunities for perpetrators to sexually abuse children. The result that living in single parent families was a significant risk factor of witnessing parental conflict was in line with the finding of a study about intimate partner violence in Vietnam indicating that conflicts still occurred after a separation or divorce (Jansen et al., 2016). Perhaps a considerable part of single parenthood in our sample concerned separated or divorced parents, or parents in the process of a separation, which would increase the risk of witnessing parental conflicts. Regarding neglect, it is interesting that neither socioeconomic nor employment status predicted neglect experiences, but living situation played an important role. The overload single parents may experience from taking care of the household, providing family income, and being responsible for their child's education, physical, and emotional needs on their own, and may increase the likelihood of child neglect. This finding is partly consistent with a meta-analysis that found that in addition to unemployment, large family size, and low SES, single parenthood was related to neglect (Stith et al., 2009). However, only univariate associations were addressed in this meta-analysis.

SES and parental employment status were only associated with emotional abuse experience. In contrast with previous literature that low SES is a risk factor for emotional abuse (Black, Smith Slep, \& Heyman, 2001), we found that low SES and parental unemployment lowered the likelihood of experiencing emotional abuse in the past year and during lifetime, respectively, although the effect for SES in particular was very small. In our previous article on the consequences of child maltreatment, we also found that emotional abuse was associated with better academic performance (Tran, van Berkel, et al., 2017). The practice of "Tiger parenting" may play a role in this discrepancy (Chua, 2011). This parenting style, which favors harsh discipline and puts pressure on children to achieve high academic results and gain honor for the family, has been found to be common in Asian families (Kim et al., 2013; Nguyen et al., 2014) and may be reflected in experiences of emotional 
abuse. Parents with low SES or unemployment may put lower expectations of academic performance on their children than parents with higher SES or better employment status, so their children may report fewer emotional abuse experiences. This phenomenon, its risk factors, and consequences need more exploration in future research.

Some of the discrepancies between our findings and those from previous meta-analyses and reviews could be explained by the difference in societies and cultures. Most of the previous studies were conducted in Western highincome countries, where there may be better social programs, a larger and more skilled social service workforce, and less harsh discipline acceptance than in a limited resource country such as Vietnam. Another explanation could be that in many previous studies, risk factors were examined independently from other factors, so it was impossible to control for the effects of other factors. In the current study, we tested multivariate effects of risk factors, thus controlling for the other risk factors.

Some limitations should be mentioned. First, this is a cross-sectional study, so the chronological order between child and family factors and child maltreatment could not be specified. Nevertheless, the chronological order is obvious for some factors including gender, age, ethnicity, and war experiences of fathers. Even in case the chronological order is unclear, knowledge on associations between these factors and child maltreatment is still valuable for designing targeted intervention programs. Second, this study was conducted in Northern Vietnam only, although there is no clear evidence on differences in parenting practice between the North and the South, we should be cautious in generalizing these findings to the whole country. Third, the Emotional Abuse scale and the measure for war veteran status of fathers are based on only one single item, which limits the validity of these measures. However, a meta-analysis about emotional abuse by Stoltenborgh et al. did not find an association between the number of items (from 1 to 20 items) for the emotional abuse scale and the prevalence of emotional abuse (Stoltenborgh, Bakermans-Kranenburg, Alink, \& van IJzendoorn, 2012). A more extensive and detailed measure on traumatic war experiences administered directly to fathers could make it possible to examine the association between war experience and child maltreatment better. In addition, other factors such as social cohesion among soldiers or social support after the war could be taken into account as possible protective factors.

In sum, among all explored factors, single parenthood was associated with most types of child maltreatment including sexual abuse, witnessing parental conflict, neglect, and multiple types of child maltreatment. Therefore, single parent families should be a prioritized target population in prevention/early intervention programs. The next factor most frequently associated with child 
maltreatment was child age. Lifetime maltreatment experiences increased with age. This confirms the advantage of early intervention on child maltreatment, so additional maltreatment experiences can be prevented. Our findings also show that boys were at higher risk of experiencing sexual abuse and physical abuse than girls. More studies on these differences and on whether they are specific to East Asian cultures could clarify the underlying mechanisms so that intervention programs for these types of child maltreatment could become gender specific, and therefore better adjusted to the specific problems for boys and girls. Last but not least, more studies on emotional abuse and "Tiger parenting" in Vietnam could clarify the association of emotional abuse with high SES and parental employment.

\section{Acknowledgments}

The authors also would like to express their thanks to Dr. Vu Cong Nguyen, deputy director, and Dr. Nguyen Thanh Liem, former deputy director of Institute of Population, Health and Development for their support and their valuable advice on the VPM-2014 study.

\section{Declaration of Conflicting Interests}

The author(s) declared no potential conflicts of interest with respect to the research, authorship, and/or publication of this article.

\section{Funding}

The author(s) disclosed receipt of the following financial support for the research, authorship, and/or publication of this article: They appreciate financial support of the Netherlands Fellowship Programmes to Nhu K. Tran (NFP-PhD.12/547) and the Netherlands Organization for Scientific Research to Lenneke R. A. Alink (VIDI Grant no. 016.145.360) and to Marinus H. van IJzendoorn (NWO SPINOZA prize).

\section{References}

Alink, L. R., Euser, S., van IJzendoorn, M. H., \& Bakermans-Kranenburg, M. J. (2013). Is elevated risk of child maltreatment in immigrant families associated with socioeconomic status? Evidence from three sources. International Journal of Psychology, 48, 117-127. doi:10.1080/00207594.2012.734622

Ang, R. P., \& Goh, D. H. (2006). Authoritarian parenting style in Asian societies: A cluster-analytic investigation. Contemporary Family Therapy, 28, 131-151.

Beazley, H., Bessell, L., Ennew, J., \& Waterson, R. (2006). What children say: Results of comparative research on physical and emotional Punishment of children in Southeast Asia, East Asia and the Pacific. Bangkok: Save the Children.

Belsky, J. (1980). Child maltreatment: An ecological integration. American Psychologist, 35, 320-335. doi:10.1037/0003-066X.35.4.320 
Belsky, J. (1993). Etiology of child maltreatment: A developmental ecological analysis. Psychological Bulletin, 114, 413-434. doi:10.1037/0033-2909.114.3.413

Black, D. A., Heyman, R. E., \& Smith Slep, A. M. (2001). Risk factors for child sexual abuse. Aggression and Violent Behavior, 6, 203-229. doi:10.1016/S13591789(00)00023-9

Black, D. A., Smith Slep, A. M., \& Heyman, R. E. (2001). Risk factors for child psychological abuse. Aggression and Violent Behavior, 6, 189-201. doi:10.1016/ S1359-1789(00)00022-7

Bronfenbrenner, U. (1979). The ecology of human development. Cambridge, MA: Harvard university press.

Capaldi, D. M., Knoble, N. B., Shortt, J. W., \& Kim, H. K. (2012). A systematic review of risk factors for intimate partner violence. Partner Abuse, 3, 231-280. doi:10.1891/1946-6560.3.2.231

Chua, A. (2011). Battle hymn of the tiger mother. London, England: Bloomsbury.

Douglas, E. M., \& Straus, M. A. (2006). Assault and injury of dating partners by university students in 19 countries and its relation to corporal punishment experienced as a child. European Journal of Criminology, 3, 293-318. doi:10.1177/1477370806065584

Euser, S., Alink, L. R., Pannebakker, F., Vogels, T., Bakermans-Kranenburg, M. J., \& van IJzendoorn, M. H. (2013). The prevalence of child maltreatment in the Netherlands across a 5-year period. Child Abuse \& Neglect, 37, 841-851. doi:10.1016/j.chiabu.2013.07.004

Fang, X., Fry, D. A., Brown, D. S., Mercy, J. A., Dunne, M. P., Butchart, A. R., . . . Swales, D. M. (2015). The burden of child maltreatment in the East Asia and Pacific region. Child Abuse \& Neglect, 42, 146-162. doi:10.1016/j. chiabu.2015.02.012

Gibbs, D. A., Martin, S. L., Kupper, L. L., \& Johnson, R. E. (2007). Child maltreatment in enlisted soldiers' families during combat-related deployments. Journal of the American Medical Association, 298, 528-535. doi:10.1001/jama.298.5.528

Graham, J. W., Olchowski, A. E., \& Gilreath, T. D. (2007). How many imputations are really needed? Some practical clarifications of multiple imputation theory. Prevention Science, 8, 206-213. doi:10.1007/s11121-007-0070-9

Guilmoto, C. Z. (2012). Son preference, sex selection, and kinship in Vietnam. Population and Development Review, 38, 31-54. doi:10.1111/j.1728-4457.2012.00471.x

Jansen, H. A., Nguyen, T. V., \& Hoang, T. A. (2016). Exploring risk factors associated with intimate partner violence in Vietnam: Results from a cross-sectional national survey. International Journal of Public Health, 61, 923-934. doi:10.1007/s00038-016-0879-8

Ji, K., Finkelhor, D., \& Dunne, M. (2013). Child sexual abuse in China: A metaanalysis of 27 studies. Child Abuse \& Neglect, 37, 613-622. doi:10.1016/j. chiabu.2013.03.008

Kim, S. Y., Wang, Y., Orozco-Lapray, D., Shen, Y., \& Murtuza, M. (2013). Does “tiger parenting" exist? Parenting profiles of Chinese Americans and adolescent developmental outcomes. Asian American Journal of Psychology, 4, 7-18. doi:10.1037/ a0030612 
Leone, J. M. (2017). Drawing invisible wounds: War comics and the treatment of trauma. Journal of Medical Humanities. Advance online publication. doi:10.1007/ s10912-017-9442-8

Merckelbach, H., Smeets, T., \& Jelicic, M. (2009). Experimental simulation: Type of malingering scenario makes a difference. Journal of Forensic Psychiatry \& Psychology, 20, 378-386. doi:10.1080/14789940802456686

Mikton, C., \& Butchart, A. (2009). Child maltreatment prevention: A systematic review of reviews. Bulletin of the World Health Organization, 87, 353-361. doi:10.2471/BLT.08.057075

Miller, K. E., \& Rasmussen, A. (2010). War exposure, daily stressors, and mental health in conflict and post-conflict settings: Bridging the divide between traumafocused and psychosocial frameworks. Social Science \& Medicine, 70, 7-16. doi:10.1016/j.socscimed.2009.09.029

Nguyen, H. T. (2006). Child maltreatment in Vietnam: Prevalence and associated mental and physical health problems. Brisbane: Queensland University of Technology.

Nguyen, H. T., Chang, P. P., \& Loh, J. M. (2014). The psychology of Vietnamese tiger mothers: Qualitative insights into the parenting beliefs and practices of Vietnamese-Australian mothers. Journal of Family Studies, 20, 48-65. doi:10.5172/jfs.2014.20.1.48

Nguyen, P. V. (2008). Perceptions of Vietnamese fathers' acculturation levels, parenting styles, and mental health outcomes in Vietnamese American adolescent immigrants. Social Work, 53, 337-346.

Rentz, E. D., Marshall, S. W., Loomis, D., Casteel, C., Martin, S. L., \& Gibbs, D. A. (2007). Effect of deployment on the occurrence of child maltreatment in military and nonmilitary families. American Journal of Epidemiology, 165, 1199-1206. doi:10.1093/aje/kwm008

Saile, R., Ertl, V., Neuner, F., \& Catani, C. (2014). Does war contribute to family violence against children? Findings from a two-generational multi-informant study in Northern Uganda. Child Abuse \& Neglect, 38, 135-146. doi:10.1016/j. chiabu.2013.10.007

Sedlak, A. J., Mettenburg, J., Basena, M., Peta, I., Mcpherson, K., \& Greene, A. (2010). Fourth National Incidence Study of child abuse and neglect (NIS-4). Washington, DC: U.S. Department of Health and Human Services.

Smith, M. (2004). Parental mental health: Disruptions to parenting and outcomes for children. Child \& Family Social Work, 9, 3-11.

Stith, S. M., Liu, T., Davies, L. C., Boykin, E. L., Alder, M. C., Harris, J. M., . . . Dees, J. E. M. E. G. (2009). Risk factors in child maltreatment: A meta-analytic review of the literature. Aggression and Violent Behavior, 14, 13-29. doi:10.1016/j.avb.2006.03.006

Stoltenborgh, M., Bakermans-Kranenburg, M. J., Alink, L. R., \& van IJzendoorn, M. H. (2012). The universality of childhood emotional abuse: A meta-analysis of worldwide prevalence. Journal of Aggression, Maltreatment \& Trauma, 21, 870890. doi:10.1080/10926771.2012.708014

Stoltenborgh, M., Bakermans-Kranenburg, M. J., Alink, L. R., \& van IJzendoorn, M. H. (2014). The prevalence of child maltreatment across the globe: Review of a series of meta-analyses. Child Abuse Review, 24, 37-50. doi:10.1002/car.2353 
Straus, M. A., Hamby, S. L., Finkelhor, D., Moore, D. W., \& Runyan, D. (1998). Identification of child maltreatment with the Parent-Child Conflict Tactics Scales: Development and psychometric data for a national sample of American parents. Child Abuse \& Neglect, 22, 249-270. doi:10.1016/S01452134(97)00174-9

Tabachnick, B. G., \& Fidell, L. S. (2012). Using multivariate statistics (6th ed.). Boston, MA: Pearson.

Tran, N. K., Alink, L. R., van Berkel, S. R., \& van IJzendoorn, M. H. (2017). Child maltreatment in Vietnam: Prevalence and cross-cultural comparison. Journal of Aggression, Maltreatment \& Trauma, 26, 211-230. doi:10.1080/10926771.2016 .1250851

Tran, N. K., van Berkel, S. R., van IJzendoorn, M. H., \& Alink, L. R. A. (2017). The association between child maltreatment and emotional, cognitive, and physical health functioning in Vietnam. BMC Public Health, 17, Article 332. doi:10.1186/ s12889-017-4258-z

van Buuren, S. (2012). Flexible imputation of missing data. Boca Raton: CRC press. Wildman, R. W., \& Wildman, R. W., II. (1999). The detection of malingering. Psychological Reports, 84, 386-388. doi:10.2466/pr0.1999.84.2.386

\section{Author Biographies}

Nhu K. Tran is researcher at the Institute of Population, Health and Development (PHAD) in Vietnam. Her research focuses on child maltreatment and mental health. She has been involved in projects on parenting skills for parents of children with mental health problems and self-help skills for adults with mental health problems. She has a background in pharmacy and public health, holds a master of public health, and a $\mathrm{PhD}$ in child and family studies at Leiden University for which she received a grant from the Netherlands Fellowship Programmes.

Sheila R. van Berkel, PhD, is a postdoctoral researcher at the center for Child and Family Studies at Leiden University. She is involved in several studies of the research cluster Child maltreatment. With these studies, she investigates differences between maltreating and nonmaltreating parents to explore risk factors and processes that lead to maltreatment, with a specific focus on parental stress regulation, improving effectiveness of decisions concerning out-of-home-placements and the third National Prevalence studies of Maltreatment of youth in the Netherlands (NPM-2017). In addition, she collaborates with the Faculty of Governance and Global Affairs in a study on fatal violence in the Netherlands.

Marinus H. van IJzendoorn, $\mathrm{PhD}$, is a professor of Child and Family Studies at Leiden University and professor of Human Development at Erasmus University Rotterdam. His work focusses on the social, psychological, and neurobiological determinants of parenting and child development. He initiated and designed the first National Prevalence study of Maltreatment of youth in the Netherlands (NPM2005) and co-developed the Video-feedback Intervention to promote Positive Parenting (VIPP) with applications in various clinical populations. He has been 
highly influential in research on attachment theory, child maltreatment, and differential susceptibility and belongs to the $1 \%$ most cited researchers in Psychiatry and Psychology worldwide.

Lenneke R. A. Alink, $\mathrm{PhD}$, is professor of Forensic Family Studies at Leiden University and director of the research cluster Child maltreatment. Her research focuses on the prevalence, risk factors, predictors, and consequences of child maltreatment, using a multidisciplinary approach, including neurobiological factors as well as socioeconomic and family processes. She has performed the second National Prevalence study of Maltreatment of youth in the Netherlands in 2010 and is currently working on the third prevalence study in the Netherlands. She has published numerous articles and book chapters addressing child maltreatment issues. 